\title{
Chylothorax Associated with Subcutaneous Cellulitis Caused by Chylous Fluid Leakage in a Dog
}

\author{
Raphael Simões Vieira', Francisco Cláudio Dantas Mota르, Sofia Borin-Crivellenti ${ }^{3}$ \& Michelle Oblak ${ }^{4}$
}

\begin{abstract}
Background: Chylothorax is the accumulation of lymphatic fluid associated with molecules coming from digestion process (chyle) in the thorax. In this case report, we describe a canine patient with chylothorax that presented with an unusual clinical presentation: subcutaneous swelling due to chylous fluid leakage through the thoracic inlet.

Case: A 4-year-old spayed female canine, Giant Schnauzer, presented to the Ontario Veterinary College Health Sciences Centre for evaluation and treatment of bilateral subcutaneous masses and edema on the ventral thorax. The patient was originally evaluated by the primary care veterinarian and aspiration and cytology of the masses resulted in a large volume of fluid with low cellularity, lightly basophilic. Due to the appearance of the fluid, triglyceride levels were performed and was $7.95 \mathrm{mmol} / \mathrm{L}$ while the peripheral level was $0.5 \mathrm{mmol} / \mathrm{L}$ (ref: $0.2-1.3 \mathrm{mmol} / \mathrm{L}$ ). Based on the cytology and triglyceride levels, chylous fluid was suspected to be the cause of the swelling and edema. Over the following 10 months, the swelling progressed to bilateral tumors of approximately $15 \mathrm{~cm}$ in the lateral thorax and surrounding edematous tissues on the ventral thorax. The patient was referred for evaluation. On presentation the patient was normal aside from the described thoracic swellings and decreased lung sounds on auscultation. Thoracic ultrasound revealed bilateral pleural effusion. Thoracentesis resulted in approximately $1,200 \mathrm{~mL}$ of chylous fluid. Thoracic computed tomography (CT) and lymphangiography through popliteal injection revealed bilaterally in the soft tissues of the axillary regions, and extending along the right thoracic wall, a moderate amount of fluid dense material. The lymphangiogram revealed two sites of rupture of lymphatic vessels, in the caudal thorax (right sided), and in the cranial mediastinum/right thoracic inlet. Based on the findings, a video-assisted thoracic duct ligation and cisterna chyli ablation were performed. A PleuralPort was placed at the time of surgery for percutaneous drainage of residual fluid. Initially the subcutaneous swelling/masses were noted to have resolved and the patient was discharge 3 days postoperatively with minimal residual thoracic fluid at the time of discharge. Five days after discharge, the patient was returned because the owners complained of not being able to perform the thoracic drainage through the PleuralPort. Based on the physical exam and radiography, the suspicion of PleuralPort obstruction or kink was raised. Thoracocentesis drained $2.5 \mathrm{~L}$ of apparently chylous fluid. On the next day, the patient returned for replacement of the port. Eighteen days after the replacement of the port, the patient presented again due to concerns about an inability to drain the pleural port, and that the patient respiration was becoming more laborious, especially during the night. Due to the continued presence of chylous effusion, the owners were offered a repeat lymphangiogram to determine whether additional unligated branches could be contributing to the continued effusion. The owners elected humane euthanasia.

Discussion: Canine chylothorax with leakage of chylous fluid to the thoracic subcutaneous space has not been described until now. The fluid retrieved from the patient subcutaneous swellings was a chylous fluid, originated from the thorax. We hypothesized that the leakage of chylous fluid occurred through the thoracic inlet, where there is the pleural cupula, a protuberance of the pulmonary pleura, not protected by the ribs, place that could rupture easily. Because similar case was not reported, we found it important to be shared, so the veterinary scientific community can be aware of uncommon clinical presentations in patients with chylothorax.
\end{abstract}

Keywords: chylothorax, chylous cellulites, canine chylothorax. 


\section{INTRODUCTION}

Chylothorax is the accumulation of lymphatic fluid associated with molecules coming from digestion process (chyle) in the thorax. The etiology of this disease in domestic animals is due to rupture or increase in the pressure of major lymphatic vessels, typically the thoracic duct. The most common causes in veterinary patients are due to cardiac disease, compressive tumors on the region of the thoracic duct, thrombi or congenital, idiopathic and iatrogenic abnormalities [2]. Symptoms include tachypnea, exercise intolerance, laborious breathing, dyspnea and reduced appetite [5].

In this case report, we describe a canine patient with chylothorax that presented with an unusual clinical presentation: subcutaneous swelling due to chylous fluid leakage through the thoracic inlet.

\section{CASE}

A 4-year-old spayed female canine, Giant Schnauzer, presented to the Ontario Veterinary College Health Sciences Centre for evaluation and treatment of bilateral subcutaneous masses and edema on the ventral thorax. The dog initially presented to the primary care veterinarian for a mass in the thoracic inlet. The patient was originally evaluated by the primary care veterinarian and aspiration and cytology of the masses resulted in a large volume of fluid with low cellularity, lightly basophilic with a white blood cell count of $1.3 \times 10^{\wedge} 9 / \mathrm{L}$ and a total protein value of $26 \mathrm{~g} / \mathrm{L}$. Due to the appearance of the fluid, triglyceride levels were performed and was $7.95 \mathrm{mmol} / \mathrm{L}$ while the peripheral level was $0.5 \mathrm{mmol} / \mathrm{L}$ (ref: 0.2-1.3 mmol/L). Based on

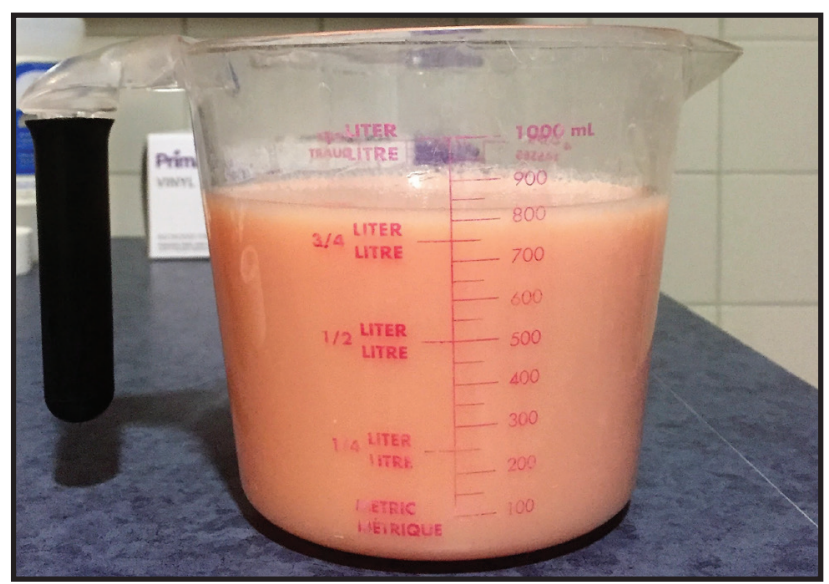

Figure 1. Chyle drained from the thorax through thoracocentesis during patient first consult. the cytology and triglyceride levels, chylous fluid was suspected to be the cause of the swelling and edema. Over the following 10 months, the swelling progressed to bilateral tumors of approximately $15 \mathrm{~cm}$ in the lateral thorax and surrounding edematous tissues on the ventral thorax. The patient was referred for evaluation. On presentation the patient was normal aside from the described thoracic swellings and decreased lung sounds on auscultation. An abbreviated thoracic ultrasound of the masses was consistent with subcutaneous edema with fluid pockets and bilateral pleural effusion. Thoracentesis resulted in approximately $1200 \mathrm{~mL}$ of chylous fluid (Figure 1).

Echocardiogram was normal with no evidence of cardiac disease. Thoracic computed tomography (CT) and popliteal lymphangiogram revealed bilaterally in the soft tissues of the axillary regions, and extending along the right thoracic wall, and mildly along the left thoracic wall, a moderate amount of fluid dense material (Figure 2). Bilaterally in the pleural space, there was a moderate to marked amount of fluid dense material. The lymphangiogram revealed two sites of rupture of lymphatic vessels, in the caudal thorax (right sided), and in the cranial mediastinum/right thoracic inlet (Figure 3). The imaging findings were consistent with a chylous cellulitis secondary to rupture of lymphatics in the cranial mediastinum and extension into the fascial planes and subcutaneous tissues.

Based on the findings, a video-assisted thoracic duct ligation and cisterna chyli ablation were performed. Intraoperatively a mesenteric lymphangiogram was performed with methylene blue and no extension of the methylene blue was noted cranial to the ligation. A PleuralPort ${ }^{1}$ was placed prior to closure (Figure 4).

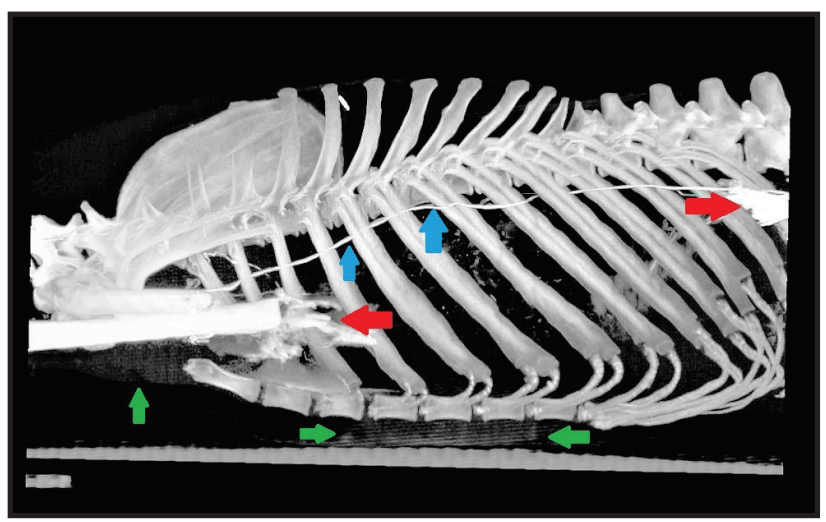

Figure 2. Tomographic popliteal lymphangiography. Blue arrows: the thoracic duct; red arrows: rupture of the thoracic duct and leakage of chylous fluid within the thorax; green arrows: leakage of the chylous fluid to the thoracic subcutaneous space. 


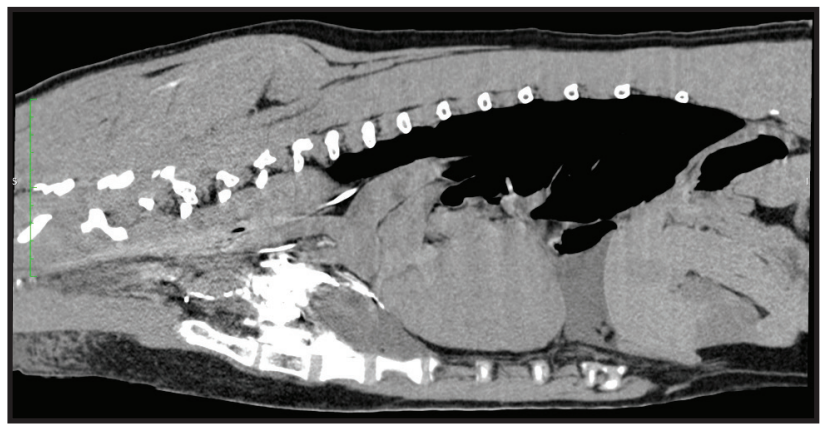

Figure 3. Tomographic lymphangiogram. Note the accumulation of contrast in the mediastinum, close to the thoracic inlet.

The patient recovered uneventfully from surgery and anesthesia. Initially the subcutaneous swelling/masses were noted to have resolved and the patient was discharge 3 days postoperatively. Five days after discharge, the patient was returned to the OVC because the owners complained of not being able to perform the thoracic drainage through the PleuralPort. Based on the physical exam and radiography, the suspicion of pleural port obstruction or kink was raised. Thoracocentesis drained 2.5 L of apparently chylous fluid. On the next day, the patient returned to the OVC for the repositioning or change of the pleural port. Under general anesthesia, it was possible to detect that the pleural port fenestrations were obstructed by fibrinous tissue, so the replacement of the entire pleural port was performed. Approximately $3 \mathrm{~L}$ of chylous fluid was drained through the new pleural port.

Eighteen days after the replacement of the pleural port, the patient presented again due to concerns about an inability to drain the pleural port, and that the patient respiration was becoming more laborious, especially during the night. On the physical exam, the patient was quiet but alert. The abnormal findings were cardiac and pulmonary sounds muffled, bilaterally. The therapeutic approach consisted in flushing the pleural port with heparinized saline, but although the saline could be pushed in, it could not be pushed back. Under sedation, thoracocentesis was performed and retrieved $4 \mathrm{~L}$ of fluid. The patient respiration improved considerably after the pleural fluid drainage. Due to the continued presence of chylous effusion, the owners were offered a repeat lymphangiogram to determine whether additional unligated branches could be contributing to the continued effusion.

Due to curative failure of all treatment proposed, and because of financial issues, the owners decided for euthanasia.

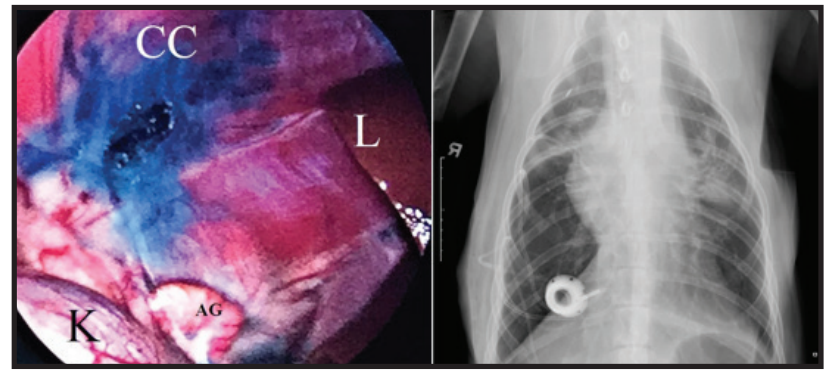

Figure 4. On the left, note the accumulation of methylene blue in the cisterna chyle (CC) and its surgical laparoscopic ablation. Cranial to the $\mathrm{CC}$ there is the liver $(\mathrm{L})$ and caudally the left kidney $(\mathrm{K})$ and the adrenal gland (AG). On the right, note the PleuralPort positioned in the dorsal caudal right thorax.

\section{DISCUSSION}

This report aimed to describe an unprecedented case of a dog with chylothorax with an unusual clinical presentation of subcutaneous swelling due to chylous fluid leakage through the thoracic inlet.

The first laboratorial analysis of the aspirated fluid from the patient thoracic subcutaneous space, revealed high concentrations of proteins and triglycerides associated with high leukocyte count, characteristic of an exudate $[7,9,16]$.

The golden standard for determining if a fluid is a chylous one, is the lipoproteins electrophoresis, that identifies specifically chylomicrons. However, if the electrophoresis is not available, liquid triglycerides measurement is considered a good and trustworthy diagnostic tool, because pleural fluid with triglycerides concentration higher than $240 \mathrm{mg} / \mathrm{dL}$ is considered chylous fluid [14]. In short, because of the laboratorial results, the fluid aspirated from the patient subcutaneous space was a chylous fluid. Additionally, another feasible and inexpensive way to differentiate chyle and pseudo-chyle could have been done in this case by promoting an overnight refrigeration and observed if a creamy layer above fluid sample would be formed, which have been associated with large amount of triglycerides and/or cholesterol [15].

The image diagnostic tools used were computed tomography (CT), tomography lymphangiography and echocardiogram. The CT made it possible to identify the thoracic organs, as well as the thoracic duct and its ruptured spots, because this equipment gives a tridimensional image with good resolution, lowering the interference of adjacent tissues overlap [12,13].

The tomographic lymphangiography, as shown in diverse papers, allowed the visualization of the anatomic regions where there was thoracic duct ruptures 
and the surgical repair $[8,10]$. The possibility of the chylothorax being caused by a cardiomyopathy, e.g. venous obstruction, congenital cardiomyopathies and pericardium effusion was discarded by echocardiogram results.

Together, image and laboratorial exams of the fluid obtained through thoracic aspiration, allowed diagnosis of chylothorax and subcutaneous cellulites in the ventral region of the thorax caused by the accumulation of fluid with the same radiopacity of the fluid causing pleural effusion.

The underlying cause for the rupture of the thoracic duct and consequent chylothorax was not found in this patient, so the chylothorax was classified as idiopathic, which according to literature, is the major form of chylothorax in dogs $[4,5]$.

What intrigues the most in this case is the fact of chylous fluid being found in the subcutaneous space, ventral to the sternal region. Until the writing of this report, another similar case was not found. The hypothesis is that the chylous fluid escaped to the thoracic subcutaneous space through the thoracic inlet, region between the first pair of ribs due to the large volume and pressure within the thoracic cavity. It is known that the most cranial portion of the pleura is called cupula, and this portion extends 2 to $3 \mathrm{~cm}$ cranial to the first rib. Then, because the pleura is a membranous non-rigid structure, there is the possibility that, due to the chylous fluid pressure, the pleural cupula ruptured, allowing the outflow of chyle to the subcutaneous thoracic region [3].
The treatments included in this case were thoracoscopically thoracic duct ligation, laparoscopically cisterna chyli ablation and positioning of a pleural port, once according to literature the thoracic duct ligation together with cisterna chyli ablation is one of the surgical treatments most curative for chylothorax, with success rates described as higher as $88 \%[1,6]$. Besides those techniques, others surgical interventions are described in the literature, among them thoracic omentalization, thoracic duct embolization, pericardiectomy, among others. Even that, it is important to point that none of them guarantee solving totally the disease. The causes of failure to the treatment of thoracic duct ligation and cisterna chyli ablation is not well elucidated, but can to be related to the new lymphatic vessels formation, or failure to identify all points of the thoracic duct ruptures [11].

The clinical presentation of our patient was exceptional, and similar cases were not found in medical literature until the publication of this paper. The final diagnosis was chylothorax with subcutaneous edema due to the leakage of chylous fluid from the thorax, through the pleural cupula. Although this clinical presentation is being written for the first time, veterinary clinicians should be aware to this occurrence, offering the best diagnosis and treatment to the patient.

\section{MANUFACTURER}

${ }^{1}$ Norfolk Vet Products. Skokie, IL, USA.

Declaration of interest. The authors report no conflicts of interest. The authors alone are responsible for the content and writing of the paper.

\section{REFERENCES}

1 Allman D.A., Radlinsky M.G., Ralph A.G. \& Rawlings C.A. 2010. Thoracoscopic thoracic duct ligation and thoracoscopic pericardectomy for treatment of chylothorax in dogs. Veterinary Surgery. 39(1): 21-27.

2 Birchard S.J., McLoughlin M.A. \& Smeak D.D. 1995. Chylothorax in the dogs and cat: a review. Lymphology. 28(2): 64-72.

3 Finley D.J. \& Rusch V.W. 2011. Anatomy of the pleura. Thoracic Surgery Clinics. 21(2): 173-175.

4 Fossum T.W., Birchard S.J. \& Jacobs R.M. 1986. Chylothorax in 34 dogs. Journal of the American Veterinary Medical Association. 188(11): 1315-1318.

5 Fossum T.W., Mertens M.M., Miller M.W., Peacock J.T., Saunders A., Gordon S., Pahl G., Makarski L.A., Bahr A. \& Hobson P.H. 2004. Thoracic duct ligation and pericardectomy for treatment of idiopathic chylothorax. Journal of Veterinary Internal Medicine. 18(3): 307-310.

6 Hayashi K., Sicard G., Gellasch K., Frank J.D., Hardie R.J. \& McAnulty J.F. 2005. Cisterna chyli ablation with thoracic duct ligation for chylothorax: Results in eight dogs. Veterinary Surgery. 34(5): 519-523.

7 Heffner J.E., Sahn S.A. \& Brown L.K. 2002. Multilevel likelihood ratios for identifying exudative pleural effusions. Chest 121(6): 1916-1920. 
8 Kawasaki R., Sugimoto K., Fujii M., Miyamoto N., Okada T., Yamaguchi M. \& Sugimura K. 2013. Therapeutic effectiveness of diagnostic lymphangiography for refractory postoperative chylothorax and chylous ascites: Correlation with radiologic findings and preceding medical treatment. American Journal of Roentgenology. 201(3): 659-666.

9 Maldonado F., Hawkins F.J., Daniels C.E., Doerr C.H., Decker P.A. \& Ryu J.H. 2009. Pleural fluid characteristics of chylothorax. Mayo Clinic Proceedings. 84(2): 129-133.

10 Sachs P.B., Geisinger M.A., Risius B. \& Lammert G.K. 1991. Diagnosis and Localization of the Thoracic Duct: usefulness of Lymphangiography and CT. American Roentgen Ray Society. 157(4): 703-705.

11 Sicard G.K., Waller K.R. \& McAnulty J.F. 2005. The effect of cisterna chyli ablation combined with thoracic duct ligation on abdominal lymphatic drainage. Veterinary Surgery. 34(1): 64-70.

12 Singh A., Brisson B. \& Nykamp S. 2012. Idiopathic chylothorax: pathophysiology, diagnosis, and thoracic duct imaging. Compendium on Continuing Education for the Practising Veterinarian. 34(8): E2.

13 Singh A., Brisson B.A., Nykamp S. \& O'Sullivan M.L. 2011. Comparison of computed tomographic and radiographic popliteal lymphangiography in normal dogs. Veterinary Surgery. 40(6): 762-767.

14 Thaler M.A., Bietenbeck A., Schulz C. \& Luppa P.B. 2017. Establishment of triglyceride cut-off values to detect chylous ascites and pleural effusions. Clinical Biochemistry. 50(3): 134-138.

15 Thrall M.A., Weise G., Allison R.W. \& Campbell T.W. 2012. Veterinary Hematology and Clinical Chemistry. 2nd edn. Ames: John Wiley \& Sons, 776p.

16 Tyler R.D. \& Cowell R.L. 1989. Evaluation of pleural and peritoneal effusions. Veterinary Clinics of North America: Small Animal Practice. 19(4): 743-768. 